\title{
QIDANTONGMAI PROTECTS ENDOTHELIAL CELLS AGAINST HYPOXIA-INDUCED DAMAGE THROUGH REGULATING THE SERUM VEGF-A LEVEL
}

\author{
Bing Wang ${ }^{1,2 \#}$, Wen Wang ${ }^{1 \#}$, Feng $\mathrm{Li}^{1 \#}$, Zongren Wang ${ }^{1}$, Jing $\mathrm{Ma}^{1 *}$, Gang Zhao ${ }^{2 *}$ \\ ${ }^{1}$ Department of Traditional Chinese Medicine, ${ }^{2}$ Department of Neurology, Xijing Hospital, The \\ Fourth Military Medical University, Xi'an, Shanxi 710032, People's Republic of China. \\ *Email: Jingma@fmmu.edu.cn, zhaogang@,fmmu.edu.cn
}

\begin{abstract}
Qidantongmai (QDTM) is a Traditional Chinese Medicine (TCM) preparation that has long been used in folk medicine for the treatment of cardiovascular diseases. However, the underlying mechanisms are poorly understood. The present study was designed to determine the effects of QDTM on endothelial cells under hypoxic conditions both in vitro and in vivo. Primary human umbilical vein endothelial cells (HUVECs) were isolated, pretreated with QDTM medicated serum or saline control, and then cultured under hypoxia (2\% oxygen) for $24 \mathrm{~h}$. Sprague-Dawley rats were administered $1 \mathrm{ml} / 100 \mathrm{~g}$ of QDTM or saline twice a day for 4 days and treated with hypoxia (6 hours/day, discontinuous hypoxia, $360 \mathrm{~mm} \mathrm{Hg}$ ). QDTM not only protected HUVECs from hypoxia-induced damage by significantly retaining cell viability $(P<0.05)$ and decreasing apoptosis $(P<0.05)$ in vitro, but also protected liver endothelial cells from hypoxia-induced damage in vivo. Moreover, QDTM increased the serum VEGF-A level $(P<0.05)$ in rats treated with hypoxia for 7 days but suppressed the upregulation of serum VEGF-A in rats treated with hypoxia for 14 days. QDTM is a potent preparation that can protect endothelial cells against hypoxia-induced damage. The ability of QDTM to modulate the serum VEGF-A level may play an important role in its effects on endothelial cells.
\end{abstract}

Key words: Traditional Chinese Medicine, human umbilical vein endothelial cells, hypoxia, VEGF

\section{Introduction}

Natural herbal products have been used in Traditional Chinese Medicine (TCM) for centuries, and increasing evidence supports the effectiveness of TCM in clinical therapies and animal experiments. For example, Qidantongmai (QDTM), a TCM formulation that has been used to treat brain ischemia-reperfusion damage, myocardial ischemia-reperfusion damage, atherosclerosis, thrombosis, and coronary artery diseases (Ma et al., 2000; Wang et al., 1998; Zhang et al., 2001a), is composed of Astragalus, Salvia, Angelica sinensis, Ramulus cinnamoni, and Carthamus tinctorius. All five of these TCMs are widely used to treat cardiovascular diseases, such as angina pectoris, myocardial infarction, and atherosclerosis. The present study was designed to determine the effects of QDTM on human endothelial cells and to uncover the underlying mechanisms for its actions.

Ischemia, which is characterized by decreased oxygen delivery and nutrient supply to tissues, is involved in many human diseases, such as thrombosis, atherosclerosis, myocardial infarction, and cerebral ischemia (Kanagy, 2009; Morgan, 2007). Endothelial cells are the first target in ischemia-induced hypoxia (Paternotte et al., 2008; Ten and Pinsky, 2002), resulting in the activation of endothelial cells (Paternotte et al., 2008). Thus, it is important to develop strategies for prevention and treatment of hypoxia-induced cellular and tissue damage. Although QDTM has been used to treat cardiovascular disorders for many years, the effects and mechanisms of QDTM on endothelial cells under hypoxic conditions have not been investigated.

http://dx.doi.org/10.4314/ajtcam.v9i2.5 
Vascular endothelial growth factor (VEGF) is a major regulator of endothelial proliferation and migration (Majesky, 1996). The seven members of the VEGF family are VEGF-A, VEGF-B, VEGF-C, VEGF-D, VEGF-E, VEGF-F, and PIGF. As a key target for new anti-angiogenic drugs for the treatment of both malignant and nonmalignant human diseases, VEGF exhibits multiple functions (Ellis and Hicklin, 2008). Moreover, VEGF-modulated vascular biological functions may contribute to the development and progression of several human diseases, including cardiovascular diseases, cancer, and diabetic complications. Therefore, targeting VEGF is a potentially effective approach for the treatment of ischemic disorders. Upregulation of VEGF has been observed in tumors and under various conditions such as hypoxia and wound healing (Fan et al., 2002; Mori et al., 2009). In the present study, the serum levels of VEGF-A, which is generally considered to be a blood vessel-specific angiogenesis factor, was measured to explore the mechanism underlying the protective effects of QDTM on endothelial cells.

\section{Materials and Methods}

Animals

Male Sprague-Dawley (SD) rats weighing $250 \pm 20 \mathrm{~g}$ were purchased from the Experimental Center of The Fourth Military Medical University, Xi'an, China, and housed under controlled conditions (temperature $23^{\circ} \mathrm{C} \pm 1{ }^{\circ} \mathrm{C}$, humidity $60 \% \pm 10 \%, 12-\mathrm{h} / 12-\mathrm{h}$ light/dark rhythm) and with free access to water and chow. The animal experiments were approved by the Ethics Committee for Animal Use of The Fourth Military Medical University.

\section{QTCM and medicated serum preparation}

The QDTM tablets were kindly provided by Xijing Hospital (Xi'an, China). To prepare the QDTM medicated serum preparation, SD rats were given QDTM orally at a dose of $3.24 \mathrm{~g} / \mathrm{kg}$ twice a day for 4 days. Blood samples were taken $1 \mathrm{~h}$ after the last dosing and centrifuged to prepare medicated serum. Serum was frozen at $-80{ }^{\circ} \mathrm{C}$ until use. The control serum was collected from normal rats treated with saline.

\section{Cells and cell culture}

Human umbilical vein endothelial cells (HUVECs) were isolated from fresh human umbilical veins as described previously, with some modifications (Fan et al., 2002). The study was reviewed and approved by the Ethics Review Board of the Fourth Military Medical University, and all participants gave informed consent. Briefly, to prepare HUVECs, human umbilical veins were harvested from umbilical cords under sterile conditions, flushed with phosphate-buffered saline (PBS), filled with PBS containing 0.2\% collagen II (from Sigma-Aldrich Company, St. Louis, MO), and then incubated for 20 mins at $37{ }^{\circ} \mathrm{C}$. The HUVECs were then collected and washed with PBS. The isolated primary HUVECs were maintained in $2 \%$ gelatin-coated tissue culture plates in complete growth medium 199 supplemented with $20 \%$ fetal bovine serum, $10 \mu \mathrm{g} / \mathrm{ml}$ of endothelial cell growth factor (from Gibco BRL, Life Technologies, New York, USA), $10 \mathrm{mg} / \mathrm{ml}$ of heparin, and penicillin-streptomycin $\left(50 \mathrm{mg} / \mathrm{ml}\right.$ each) at $37{ }^{\circ} \mathrm{C}$ in a $5 \% \mathrm{CO}_{2}$ incubator. All experiments were performed with cells between passages 4 and 8 .

\section{Immunofluorescence}

The expression level of factor VIII was detected by immunofluorescence performed as reported (Shen et al., 2010). For cell imaging, cells were cultured onto acid-washed, fibronectin-coated glass cover slips and allowed to adhere 
overnight. The expression of factor VIII was detected by using the rabbit anti-rat factor VIII antibody (Zhongshan Co., Beijing,China) and the FITC conjugated human anti-rabbit IgG (Santa Cruz, CA, USA). The slides were observed and pictures were taken under a fluorescence microscope (Olympus, Tokyo, Japan).

\section{In vitro cell viability assay}

The in vitro experiments with HUVECs included three groups: the normal control group without any treatment (NC group), the control serum group (S group), and the QDTM medicated serum group (Q group). For the S and Q groups, the HUVECs were incubated with $10 \%$ saline in medicated serum and 10\% QDTM in medicated serum, respectively, and grown at $37{ }^{\circ} \mathrm{C}$ in a humidified atmosphere with $5 \% \mathrm{CO}_{2}$ and $2 \%$ oxygen for $24 \mathrm{~h}$. Cell viability was determined by the MTT assay as described previously (Bittner et al., 2010; Perier et al., 2010). The extent of reduction of MTT to formazan within cells was quantified by the measurement of optical density at $490 \mathrm{~nm}$ with a micro-plate reader (Sigma-Aldrich, St. Louis, MO) (Wang et al., 2011a).

\section{Determination of apoptosis by flow cytometry}

Apoptosis was analyzed by flow cytometry with Hoechst 33342/PI staining. The cultured HUVECs were washed with PBS twice, resuspended in PBS, and incubated with Hoechst 33342 at $37{ }^{\circ} \mathrm{C}$ for $10 \mathrm{~min}$. Next, suspensions were centrifuged again to obtain cell pellets. Cells were stained with $1 \mathrm{ml}$ propidium iodide (PI) at $4{ }^{\circ} \mathrm{C}$ for 15 min and then detected using an Epics Altra II flow cytometer (Beckman, Los Angles, CA, USA) (Wang et al., 2011b).

\section{Determination of effects of QDTM on rats following hypoxia treatment}

A total of $30 \mathrm{SD}$ rats were randomly assigned to one of three groups: the normal control $(\mathrm{NC})(\mathrm{n}=6)$; hypoxia and saline $(H+S)(n=12)$; and hypoxia and QDTM $(H+Q)$ groups $(n=12)$. For the NC group, rats were maintained at normal pressure and normal oxygen. For the other two groups, hypoxia was induced by placing the rats into a hypobaric hypoxia cabinet (Chakkarapani et al., 2010; Fan et al., 2010) with 6 h/day discontinuous hypoxia (360 mm Hg). For the H + $\mathrm{S}$ group, $1 \mathrm{ml} / 100 \mathrm{~g}$ saline was orally administered to each rat twice a day for 4 days before the hypoxia treatment. For the $\mathrm{H}$ + Q group, $1 \mathrm{ml} / 100 \mathrm{~g}$ QDTM solution $(32.4 \mathrm{~g} / \mathrm{ml})$ was orally administered to each rat twice a day for 4 days before hypoxia treatment. At 7 and 14 days after the hypoxia treatment, six rats from each groups were sacrificed; blood samples were taken from the femoral artery and the livers were harvested and fixed in formalin for $24 \mathrm{~h}$. Sera were obtained and stored at $-80^{\circ} \mathrm{C}$ for further use.

\section{Histology analysis}

The formalin-fixed liver tissues were washed, dehydrated in a graded ethanol series, and embedded in paraffin. Sections $(5 \mu \mathrm{m})$ were cut transversely and stained with haematoxylin and eosin (HE) (Shen et al., 2010; Wang et al., 2011c) for light microscopic investigation.

\section{Transmission Electron microscopy}

HUVECs then were washed, digested, resuspended, and centrifuged (1000 rpm) and then the pellet was harvested. For electron microscopy, the pellets and livers were fixed in $30 \mathrm{ml} / \mathrm{L}$ glutaraldehyde, and then postfixed with osmium tetraoxide, dehydrated in a graded ethanol series, and embedded in epoxy resin. Samples were sectioned (50 nm), stained 
with uranyl acetate and lead citrate, and observed under a JEM-1200EX Electron Microscope TEM (Japan Electron Optics Ltd., Peabody, Japan) (Lamhonwah et al., 2010).

Determination of serum VEGF-A levels by ELISA

Serum was inactivated at $56{ }^{\circ} \mathrm{C}$ for $30 \mathrm{~min}$ and filtered with a $0.22 \mu \mathrm{m}$ filter membrane. The serum VEGF-A levels were determined using the ELISA method (Zheng et al., 2010) according to the manufacturer's instruction (Sen Xiong Corp, Shanghai, China).

\section{Statistical Analysis}

The experimental data were expressed as mean \pm standard deviation (SD). Comparisons among groups were conducted with analysis of variance (ANOVA) or an unpaired $t$-test with SPSS software (Version 11.5, Chicago, IL, USA). $P$ $<0.05$ was considered significant.

\section{Results}

Morphological analysis of HUVECs

The morphological characteristics of the untreated HUVECs were observed under an inverted phase contrast microscope. As shown in Figure 1A, typical slab stone-looking monolayer cells were observed, which is characteristic of endothelial cells. Factor VIII expression was detected by immunofluorescence. As shown in Figure 1B, factor VIII was positively stained in cultured HUVECs.

\section{Figure 1}

A

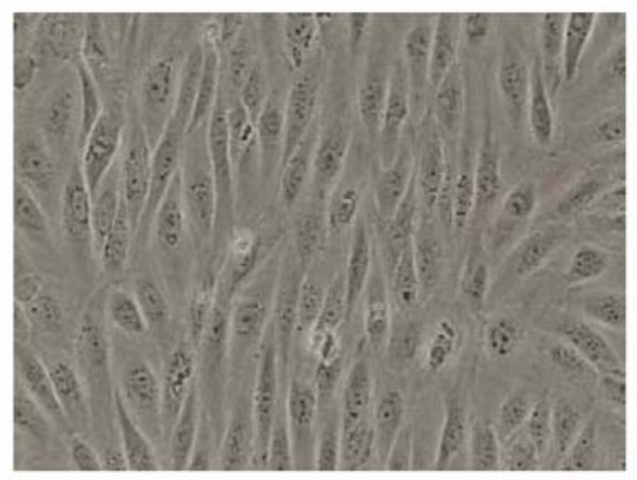

B

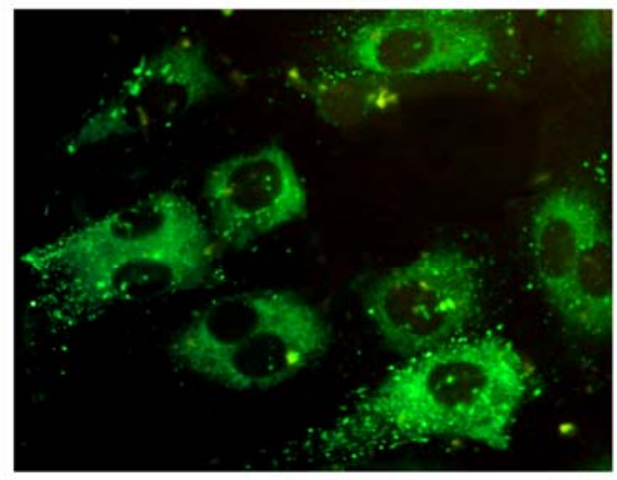

Figure 1: Cultured human umbilical vein endothelial cells (HUVECs). (A) HUVECs in confluence $(\times 100)$; (B) Factor VIII was stained by anti-factor VIII antibody and then FITC conjugated human anti-rabbit IgG (green). Images were obtained by fluorescence microscopy. 
Figure 2

A

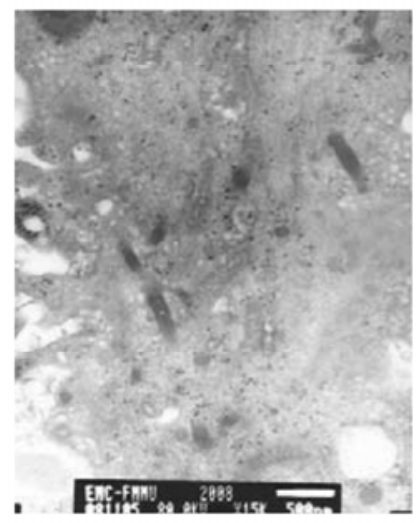

B

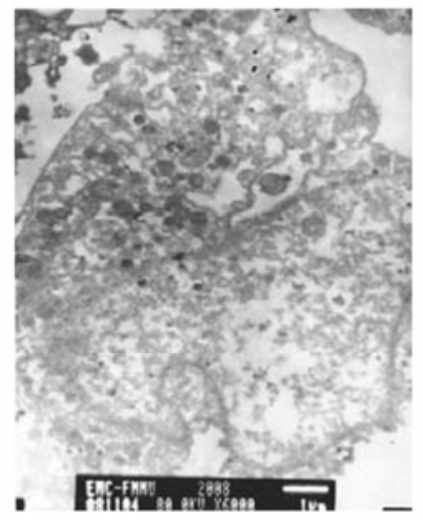

C

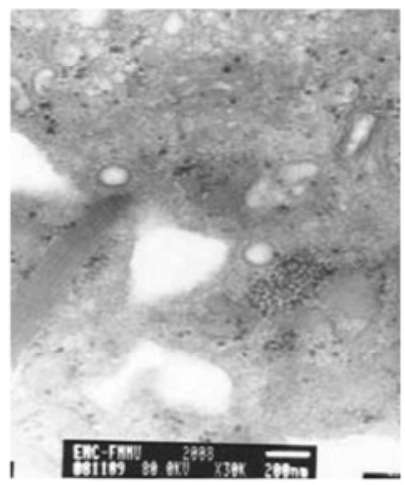

Figure 2: Transmission electron microscopy evidence that QDTM protects human umbilical vein endothelial cells (HUVECs) from hypoxia-induced damage. QDTM medicated serum from SD rats (See Methods section for detailed information) was added to cell culture medium to determine the effect of QDTM on hypoxia-treated HUVECs. Representative images are shown. (A) NC (normal control) group $(\times 200)$; (B) H + S (hypoxia + saline) group $(\times 200)$; (C) H + Q (hypoxia + QDTM treatment) group $(\times 200)$.

\section{Effects of QDTM on microarchitecture of HUVECs under hypoxia}

To evaluate whether QDTM can protect HUVECs from hypoxia stress, we observed the microarchitecture of HUVECs after hypoxia treatment using an electronic microscope. As shown in Figure 2A, typical Weible-Palade bodies were observed in HUVECs in the NC group, indicating the integrity of the microarchitecture. As expected, cell necrosis, karyolysis, and broken cell membranes were observed in the HUVECs of the S group (Figure 2B). However, Weible-Palade bodies and some membrane-like structure were observed in the HUVECs in the Q group (Fig. 2C), indicating that QDTM protected the microarchitecture of HUVECs following hypoxia treatment.

\section{Effects of QDTM on cell viability and apoptosis of HUVECs under hypoxia}

Next, we determined the effects of QDTM on cell viability and apoptosis of HUVECs. As shown in Figure 3A, the cell viability of HUVECs in the S group was significantly decreased by hypoxia treatment compared with that in the control group ( $\mathrm{n}=8, P<0.05)$. However, treatment with QDTM significantly prevented the decrease of cell viability in HUVECs compared with the saline control $(\mathrm{n}=8 ; P<0.05)$. Moreover, as shown in Figure 3B, compared with the normal control, hypoxia induced obvious apoptosis in $\mathrm{S}$ group $(\mathrm{n}=3, P<0.05)$, whereas treatment with QDTM significantly prevented apoptosis $(\mathrm{n}=3, P<0.05)$.

\section{In vivo effects of QDTM on liver and endothelial cells following hypoxia treatment}

We next investigated the in vivo effects of QDTM on the liver and endothelial cells in rats. As shown in Figure 
4A, the morphology of the liver was normal in the NC group. Hypoxia treatment for 7 days induced cell swelling in endothelial cells of central hepatic veins in the $\mathrm{H}+\mathrm{S}$ group (Figure 4B). Irregular arrangement, degeneration, and swelling were observed under the electronic microscope (Figures $4 \mathrm{~F}$ and $\mathrm{G}$ ). The changes in the microarchitecture of endothelial cells of rats treated with hypoxia for 14 days were markedly more severe (Figure 4C) than those in rats treated with hypoxia for 7 days. However, pretreatment with QDTM for 4 days partly prevented these histological alterations in rats receiving hypoxia treatment for 7 (Figures 4D and $\mathrm{H}$ ) and 14 days (Figures 4E and I).

\section{Figure 3}

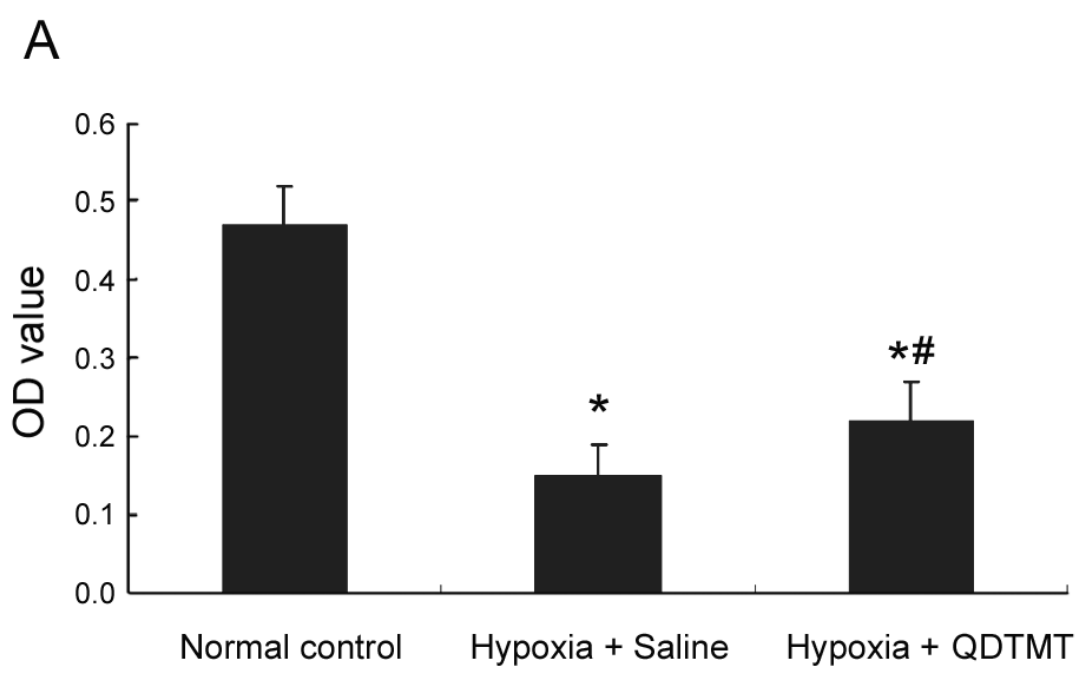

B

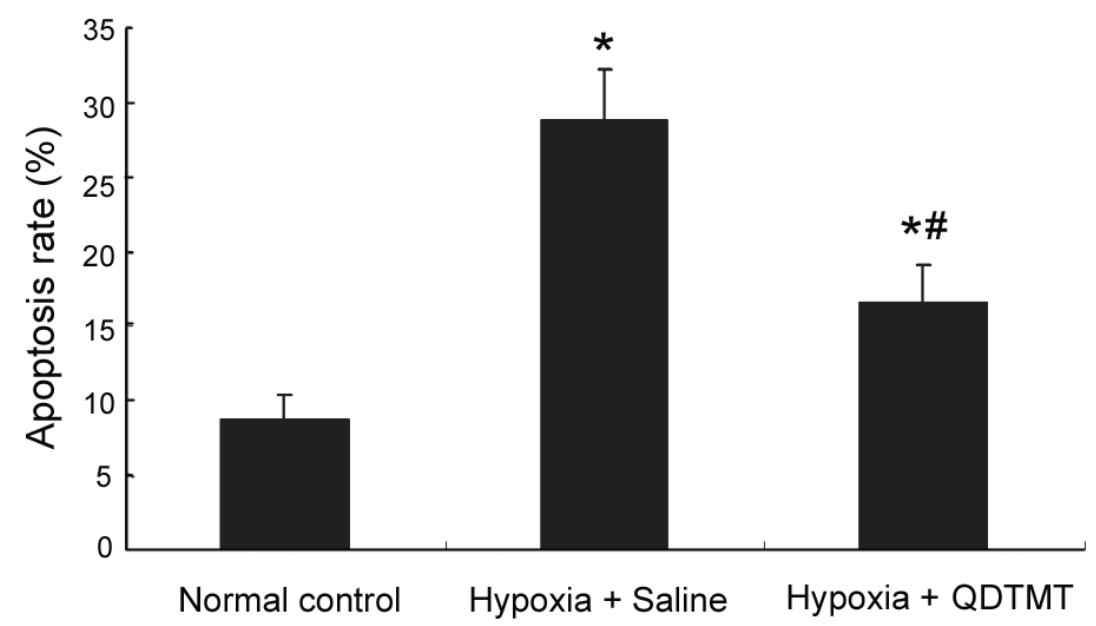

Figure 3: QDTM protects HUVECs from hypoxia-induced damage, as shown by cell viability analysis and apoptosis analysis. (A) Cell viability was determined by MTT assay. $\mathrm{n}=8,{ }^{*} P<0.05$ vs. normal control; ${ }^{\#} P<0.05$ vs. hypoxia + saline group. (B) Apoptosis rate was determined by Hoechst 33342/PI staining with flow cytometry. $\mathrm{n}=8, * P<0.05$ vs. normal control; ${ }^{\#} P<0.05$ vs. hypoxia + saline group. 


\section{Figure 4}

A

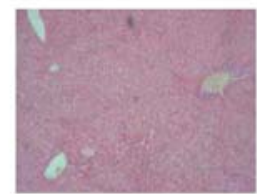

$\mathrm{F}$

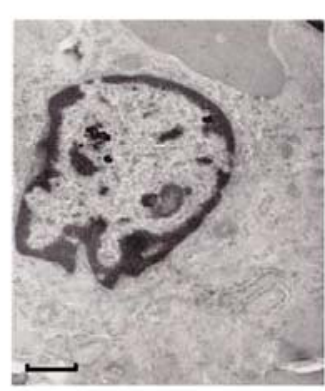

B

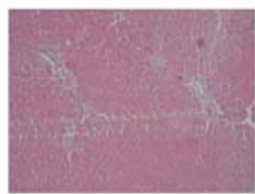

G

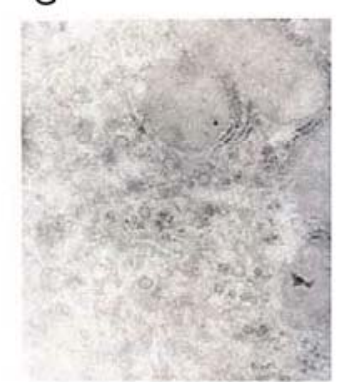

C

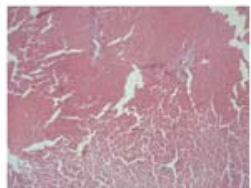

E

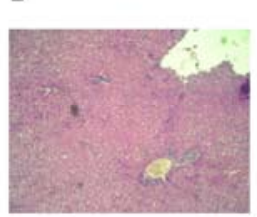

$\mathrm{H}$

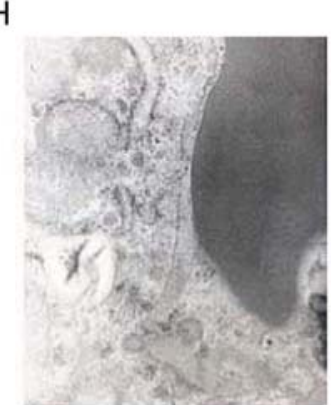

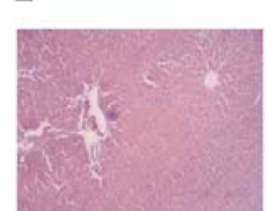

I

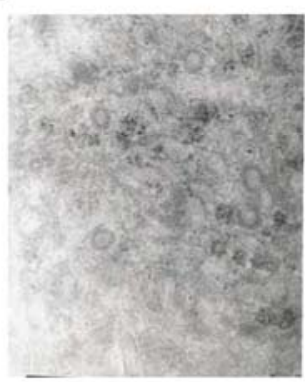

Figure 4: QDTM protects liver and endothelial cells from hypoxia-induced damage in rats as detected by histological staining and electronic microscopy. QDTM solution $(32.4 \mathrm{~g} / \mathrm{ml})$ or saline was orally administered $(1 \mathrm{ml} / 100 \mathrm{~g})$ for 4 days before hypoxia treatment ( 7 and 14 days). Tissues were fixed and then stained with haematoxylin and eosin (HE) for light microscopy (A-E) or stained with uranyl acetate and lead citrate for electronic microscopy (F-I). (A) NC group; (B, F, and G) H + S group with 7 days hypoxia treatment; (C) $\mathrm{H}+\mathrm{S}$ group with 14 days hypoxia treatment; (D, H) H + Q group with 7 days hypoxia treatment; (E, I) H + Q group with 14 days hypoxia treatment.

\section{Effects of QDTM on serum VEGF-A level}

We measured the serum VEGF-A levels in rats to determine whether QDTM could affect the expression of VEGF-A after hypoxia. Hypoxia treatment for 7 or 14 days significantly enhanced the serum VEGF-A levels compared with normal control rats $(\mathrm{n}=6, P<0.01$, Figure 5A). The pre-administration of QDTM for 4 days did not lower the serum VEGF-A level in rats on day 7, but it suppressed the upregulation of serum VEGF-A in rats on day 14 after hypoxia treatment $(\mathrm{n}=6, P<0.01$, Figure $5 \mathrm{~B})$.

\section{Discussion}

Although the underlying mechanisms for TCM's effects on various biological systems are not fully understood, the merits of their use in the treatment of chronic diseases are gradually being recognized worldwide. QDTM has been used to treat various diseases or pathological conditions, such as angina, myocardial ischemia, coronary artery disease, and other cardiovascular diseases. Our previous studies indicated that QDTM can protect the left ventricle against acute myocardial ischemia in dogs (Ma et al., 2000) and decrease the serum lipid levels in type 2 diabetes patients (Zhang et al., 2001b). All of these findings prompted us to study whether QDTM can exert protective effects against endothelial dysfunction and play a 
key role in preventing endothelial damages induced by ischemia.

\section{Figure 5}

A

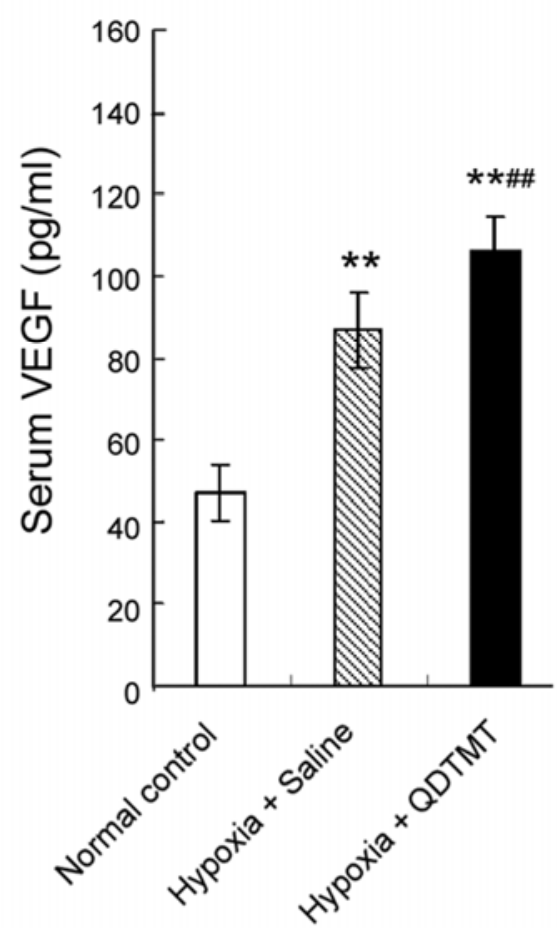

B

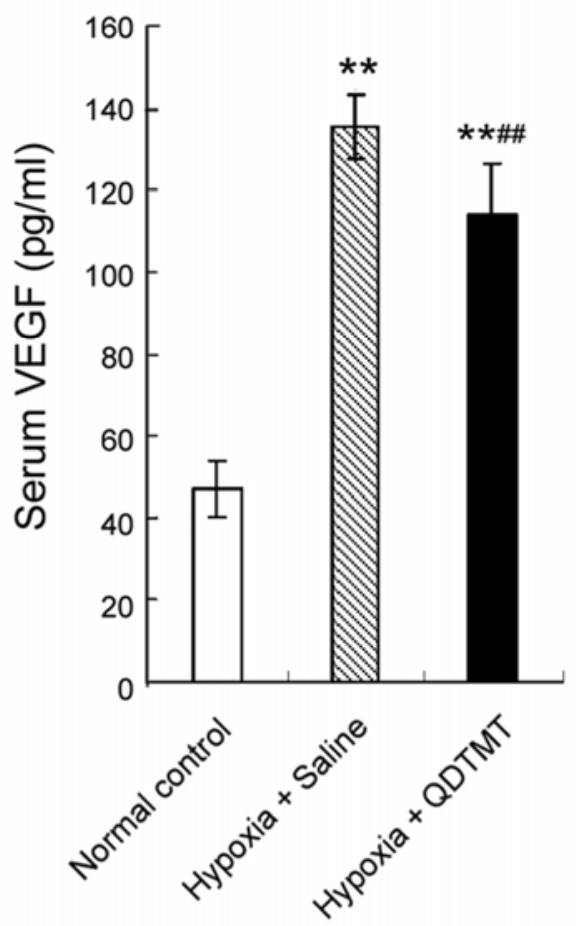

Figure 5: Serum VEGF-A levels were determined by ELISA. QDTM solution $(32.4 \mathrm{~g} / \mathrm{ml})$ or saline was orally administered $(1 \mathrm{ml} / 100 \mathrm{~g}$ ) for 4 days before hypoxia treatment (7 and 14 days). (A) Serum VEGF-A levels at 7 days after hypoxia treatment. $\mathrm{n}=6,{ }^{*} P<0.05$ vs. normal control; ${ }^{\#} P<0.05$ vs. hypoxia + saline group. (B) Serum VEGF-A levels at 14 days after hypoxia treatment. $\mathrm{n}=6,{ }^{*} P<0.05$ vs. normal control; ${ }^{\#} \mathrm{P}<0.05$ vs. hypoxia + saline group.

The present study produced three major findings. First, QDTM protected endothelial cells from hypoxia-induced damage in vitro, as shown by electronic microscopy, cell viability analysis, and apoptosis analysis in vitro. Second, oral administration of QDTM successfully ameliorated hypoxia-induced injury of hepatic veins, as shown by light and electronic microscopy. Third, hypoxia treatment for 7 and 14 days upregulated serum VEGF-A levels. Pretreatment with QDTM did not reverse the changes on day 7 but attenuated the serum VEGF-A levels by day 14. These findings may help us better understand the mechanisms of action of QDTM in treating cardiovascular diseases.

Our studies of the effects of QDTM on endothelial functions indicated that QDTM can protect endothelial cells from hypoxia-induced damage both in vitro and in vivo. These effects may result from the components in this particular combination of TCMs. Notably, there are some reports of the cardiovascular effects of Astragalus (Zhang et al., 2005; Zhang et al., 2007), Angelica sinensis (Hou et al., 2004), and Carthamus tinctorius (Ji et al., 2009; Ji et al., 2008). In the present study, our results supported the postulated protective effects of QDTM under hypoxia.

Herein, we asked the following question: How does QDTM act as an endothelial protector? A possible answer is 
that it acts via a VEGF-A-mediated mechanism. Among the seven members of the VEGF family, VEGF-A is the critical regulator of angiogenesis and vascular permeability. VEGF-A is a potent factor that can induce cell proliferation, shape change, and migration during angiogenesis (Tammela et al., 2005). Hypoxia can increase VEGF-A expression via upregulation of HIF1- $\alpha$ (Ryu et al., 2008). In our study, QDTM modulated serum VEGF-A levels both positively and negatively, depending on the time after hypoxia treatment. Previous reports have indicated that VEGF-A can be a protector (Adya et al., 2008; Dai et al., 2007) or a damager (Ferrara et al., 2003; Kopfstein et al., 2007) depending on its concentration. After injury, a low level of VEGF-A mediates angiogenesis (Benest et al., 2008), whereas a high level of VEGF-A is harmful in some cases (Kaner et al., 2000; Niimi et al., 2000). We surmise that the increase of VEGF-A levels at 7 days after hypoxia was beneficial for angiogenesis and vascular repairing; however, when the VEGF-A level reached a level that was about three-fold higher than that of normal rats at 14 days after hypoxia treatment, this level of increase was harmful. Thus, QDTM was helpful for increasing VEGF-A levels at 7 days and for downregulating VEGF-A levels at 14 days after hypoxia treatment when the VEGF-A level was too high. However, future studies are needed to determine the molecular mechanisms for this bi-phase phenomenon.

In China, QDTM is always applied with other common cardiovascular drugs such as statins, ARBs and ACEI; however, it seems that there was no available data addressing the comparison between QDTM and other currently applied drugs in cardiovascular diseases yet. In this regard, future clinical multicentric perspective study is warranted for the comparison between QDTM and other cardiovascular drugs.

In conclusion, the present study demonstrated that QDTM protected endothelial cells from hypoxia-induced damage as measured by cell viability assay, apoptosis analysis, and electronic microscopy in vitro and in vivo. Our results provide new evidence for the therapeutic value of QDTM in the treatment of cardiovascular diseases. Our data suggest that alterations of VEGF-A levels may contribute to the protective effects of QDTM.

\section{Acknowledgements}

We thank Yi Wan for statistical analysis and Yaoqing Yu for critical readings. This work was supported by the National Natural Science Foundation of China (No. 30873229), the National Department of Health Fund of China (No. 2006191001), and the Shaanxi Province Drug Administration of Traditional Chinese Medicine (No. 2007035).

\section{References}

1. Adya, R., Tan, B.K., Punn, A., Chen, J., and Randeva, H.S. (2008). Visfatin induces human endothelial VEGF and MMP-2/9 production via MAPK and PI3K/Akt signalling pathways: novel insights into visfatin-induced angiogenesis. Cardiovasc Res. 78: 356-365.

2. Benest, A.V., Harper, S.J., Herttuala, S.Y., Alitalo, K., and Bates, D.O. (2008). VEGF-C induced angiogenesis preferentially occurs at a distance from lymphangiogenesis. Cardiovasc Res. 78: 315-323.

3. Bittner, S., Bobak, N., Herrmann, A.M., G bel, K., Meuth, P., H hn, K.G., Stenner, M.P., Budde, T., Wiendl, H., and Meuth, S.G. (2010). Upregulation of K2P5. 1 potassium channels in multiple sclerosis. Ann Neurol. 68: 58-69.

4. Chakkarapani, E., Dingley, J., Liu, X., Hoque, N., Aquilina, K., Porter, H., and Thoresen, M. (2010). Xenon enhances hypothermic neuroprotection in asphyxiated newborn pigs. Ann Neurol. 68: 330-341.

5. Dai, Y., Xu, M., Wang, Y., Pasha, Z., Li, T., and Ashraf, M. (2007). HIF-1alpha induced-VEGF overexpression in bone marrow stem cells protects cardiomyocytes against ischemia. J Mol Cell Cardiol. 42: 1036-1044.

6. $\quad$ Ellis, L.M., and Hicklin, D.J. (2008). VEGF-targeted therapy: mechanisms of anti-tumour activity. Nat Rev Cancer. 8: 
579-591.

7. Fan, Y., Shen, F., Frenzel, T., Zhu, W., Ye, J., Liu, J., Chen, Y., Su, H., Young, W.L., and Yang, G.Y. (2010). Endothelial progenitor cell transplantation improves long-term stroke outcome in mice. Ann Neurol. 67: 488-497.

8. Fan, Y., Wu, D.-Z., Gong, Y.-Q., Xu, R., and Hu, Z.-B. (2002). Metabolic responses induced by thrombin in human umbilical vein endothelial cells. Biochem Biophys Res Commun. 293: 979-985.

9. Ferrara, N., Gerber, H.P., and LeCouter, J. (2003). The biology of VEGF and its receptors. Nat Med. 9: 669-676.

10. Hou, Y.Z., Zhao, G.R., Yang, J., Yuan, Y.J., Zhu, G.G., and Hiltunen, R. (2004). Protective effect of Ligusticum chuanxiong and Angelica sinensis on endothelial cell damage induced by hydrogen peroxide. Life Sci. 75: 1775-1786.

11. Ji, D.B., Zhang, L.Y., Li, C.L., Ye, J., and Zhu, H.B. (2009). Effect of Hydroxysafflor yellow A on human umbilical vein endothelial cells under hypoxia. Vascul Pharmacol. 50: 137-145.

12. Ji, D.B., Zhu, M.C., Zhu, B., Zhu, Y.Z., Li, C.L., Ye, J., and Zhu, H.B. (2008). Hydroxysafflor yellow A enhances survival of vascular endothelial cells under hypoxia via upregulation of the HIF-1 alpha-VEGF pathway and regulation of Bcl-2/Bax. J Cardiovasc Pharmacol. 52: 191-202.

13. Kanagy, N.L. (2009). Vascular effects of intermittent hypoxia. ILAR J. 50: 282-288.

14. Kaner, R.J., Ladetto, J.V., Singh, R., Fukuda, N., Matthay, M.A., and Crystal, R.G. (2000). Lung overexpression of the vascular endothelial growth factor gene induces pulmonary edema. Am J Respir Cell Mol Biol. 22: 657-664.

15. Kopfstein, L., Veikkola, T., Djonov, V.G., Baeriswyl, V., Schomber, T., Strittmatter, K., Stacker, S.A., Achen, M.G., Alitalo, K., and Christofori, G. (2007). Distinct roles of vascular endothelial growth factor-D in lymphangiogenesis and metastasis. Am J Pathol. 170: 1348-1361.

16. Lamhonwah, A.M., Bear, C.E., Huan, L.J., Chiaw, P.K., Ackerley, C.A., and Tein, I. (2010). Cystic fibrosis transmembrane conductance regulator in human muscle: Dysfunction causes abnormal metabolic recovery in exercise. Ann Neurol. 67: 802-808.

17. Ma, S.P., Wang, Z.R., and Chen, X.F. (2000). Effect of QDTMT on left ventricular in dogs with acute myocardial ischemia. J Fourth Mil Med Uinv. 21: S110-S112.

18. Majesky, M.W. (1996). A little VEGF goes a long way. Therapeutic angiogenesis by direct injection of vascular endothelial growth factor-encoding plasmid DNA. Circulation. 94: 3062-3064.

19. Morgan, B.J. (2007). Vascular consequences of intermittent hypoxia. Adv Exp Med Biol. 618: 69-84.

20. Mori, T., Sasaki, J., Kanamori, T., Aoyama, Y., and Sera, T. (2009). Hypoxia-specific upregulation of the endogenous human VEGF-A gene by hypoxia-driven expression of artificial transcription factor. Biochem Biophys Res Commun.

21. Niimi, H., Arimura, K., Jonosono, M., Hashiguchi, T., Kawabata, M., Osame, M., and Kitajima, I. (2000). VEGF is causative for pulmonary hypertension in a patient with Crow-Fukase (POEMS) syndrome. Intern Med. 39: 1101-1104.

22. Paternotte, E., Gaucher, C., Labrude, P., Stoltz, J.F., and Menu, P. (2008). Review: behaviour of endothelial cells faced with hypoxia. Biomed Mater Eng. 18: 295-299.

23. Perier, C., Bové, J., Dehay, B., Jackson - Lewis, V., Rabinovitch, P.S., Przedborski, S., and Vila, M. (2010). Apoptosis-inducing factor deficiency sensitizes dopaminergic neurons to parkinsonian neurotoxins. Ann Neurol. 68: 184-192.

24. Ryu, J., Lee, C.W., Hong, K.H., Shin, J.A., Lim, S.H., Park, C.S., Shim, J., Nam, K.B., Choi, K.J., Kim, Y.H., et al. (2008). Activation of fractalkine/CX3CR1 by vascular endothelial cells induces angiogenesis through VEGF-A/KDR and reverses hindlimb ischaemia. Cardiovasc Res. 78: 333-340.

25. Shen, W.B., McDowell, K.A., Siebert, A.A., Clark, S.M., Dugger, N.V., Valentino, K.M., Jinnah, H.A., Sztalryd, C., Fishman, P.S., and Shaw, C.A. (2010). Environmental neurotoxin-induced progressive model of parkinsonism in rats. Ann Neurol. 68: 70-80.

26. Tammela, T., Enholm, B., Alitalo, K., and Paavonen, K. (2005). The biology of vascular endothelial growth factors. Cardiovasc Res. 65: 550-563. 
27. Ten, V.S., and Pinsky, D.J. (2002). Endothelial response to hypoxia: physiologic adaptation and pathologic dysfunction. Curr Opin Crit Care. 8: 242-250.

28. Wang, P., Guan, Y.F., Du, H., Zhai, Q.W., Su, D.F., and Miao, C.Y. (2011a). Induction of autophagy contributes to the neuroprotection of nicotinamide phosphoribosyltransferase in cerebral ischemia. Autophagy. In press.

29. Wang, P., Xu, T.Y., Guan, Y.F., Tian, W.W., Viollet, B., Rui, Y.C., Zhai, Q.W., Su, D.F., and Miao, C.Y. (2011b). Nicotinamide phosphoribosyltransferase protects against ischemic stroke through SIRT1-dependent adenosine monophosphate-activated kinase pathway. Ann Neurol. 69: 360-374.

30. Wang, P., Yang, F.J., Du, H., Guan, Y.F., Xu, T.Y., Xu, X.W., Su, D.F., and Miao, C.Y. (2011c). Involvement of leptin receptor long isoform (LepRb)-STAT3 signaling pathway in brain fat mass- and obesity-associated (FTO) downregulation during energy restriction. Mol Med. 17: 523-532.

31. Wang, Z.R., Ma, S.P., and Chen, X.F. (1998). Effects of JNKST-1 on Hemodynamics and Hemorheology of Canine. Chinese Journal of Microcirculation. 2: 16-23.

32. Zhang, B.Q., Hu, S.J., Qiu, L.H., Shan, Q.X., Sun, J., Xia, Q., and Bian, K. (2005). Diphasic effects of Astragalus membranaceus BUNGE (Leguminosae) on vascular tone in rat thoracic aorta. Biol Pharm Bull. 28: 1450-1454.

33. Zhang, B.Q., Hu, S.J., Qiu, L.H., Zhu, J.H., Xie, X.J., Sun, J., Zhu, Z.H., Xia, Q., and Bian, K. (2007). Effects of Astragalus membranaceus and its main components on the acute phase endothelial dysfunction induced by homocysteine. Vascul Pharmacol. 46: 278-285.

34. Zhang, M., Ma, S.P., and Wang, Z.R. (2001a). Effect of qidan tongmai tablet on glucose and lipid metabolism in patients with diabetes mellitus type 2. Chinese journal of integrated traditional and Western medicine. 21: 825.

35. Zhang, M., Ma, S.P., and Wang, Z.R. (2001b). [Effect of qidan tongmai tablet on glucose and lipid metabolism in patients with diabetes mellitus type 2]. Zhongguo Zhong Xi Yi Jie He Za Zhi. 21: 825-827.

36. Zheng, P.P., Romme, E., Spek, P.J., Dirven, C.M.F., Willemsen, R., and Kros, J.M. (2010). Glut1/SLC2A1 is crucial for the development of the blood - brain barrier in vivo. Ann Neurol. 68: 835-844. 